\title{
Philosophiques
}

\section{Précis de Art as Performance}

\section{David Davies}

Volume 32, numéro 1, printemps 2005

Questions d'interprétation

URI : https://id.erudit.org/iderudit/011071ar

DOI : https://doi.org/10.7202/011071ar

Aller au sommaire du numéro

Éditeur(s)

Société de philosophie du Québec

ISSN

0316-2923 (imprimé)

1492-1391 (numérique)

Découvrir la revue

Citer ce document

Davies, D. (2005). Précis de Art as Performance. Philosophiques, 32(1), 207-214.

https://doi.org/10.7202/011071ar

Ce document est protégé par la loi sur le droit d'auteur. L’utilisation des services d'Érudit (y compris la reproduction) est assujettie à sa politique d'utilisation que vous pouvez consulter en ligne.

https://apropos.erudit.org/fr/usagers/politique-dutilisation/ 


\title{
Disputatio
}

\section{Précis de Art as Performance}

\author{
DAVID DAVIES \\ McGill University \\ david.davies@staff.mcgill.ca
}

Art as Performance tente à la fois de donner un meilleur fondement aux travaux récents de la philosophie analytique de l'art, et de poursuivre ces travaux de manière originale en élaborant et défendant une ontologie de l'événement artistique que je nomme «théorie de la performance». L'argument du livre présuppose un principe méthodologique concernant la manière dont nous devons procéder en ontologie de l'art, ainsi que dans la philosophie de l'art en général. Selon ce que je nomme la "contrainte pragmatique », les œuvres d'art doivent être des entités possédant le genre de propriétés que nous attribuons avec raison aux "œuvres» dans notre pratique réflexive critique et appréciative, qui sont individuées comme de telles «œuvres» le sont ou peuvent l'être, et qui possèdent les propriétés modales raisonnablement attribués aux «œuvres", dans une codification (au sens de Goodman) de cette pratique. L'œuvre est l' "unité » de la critique, de l'appréciation et de la valeur artistique - ce sur quoi portent nos jugements réflexifs critiques, appréciatifs et évaluatifs.

Dans le premier chapitre, j'élabore et défends la notion de contrainte pragmatique, à laquelle souscrivent plusieurs publications récentes sur la philosophie de l'art, dans la grande tradition analytique. Je suggère aussi qu'en vertu de la contrainte pragmatique, certains aspects de notre pratique artistique mettent en question des affirmations généralement partagées à propos de l'appréciation et de la valeur artistiques. Selon ce que je nomme la "théorie de l'art du sens commun ", les œuvres d'art sont le genre de choses dont des exemples sont accrochés sur les murs des galeries, projetés sur des écrans, joués dans des salles de concert, ou peuvent être achetés dans une librairie; et apprécier une œuvre d'art consiste à se livrer à l'expérience d'une rencontre immédiate avec un exemple de l'œuvre. La valeur artistique réside alors dans la qualité des expériences ainsi obtenues. Cette vision des choses est largement "empiriste ", si l'on tient compte du fait qu'une appréciation et une évaluation artistiques nécessitent une connaissance minimale des différents aspects de l'histoire de la production d'une œuvre, qui ne peut être déterminée sur la base de l'examen d'un seul de ses exemples - voilà ce à quoi conduit la nature "immédiate» de l'expérience nécessaire de la rencontre.

Les aspects de notre pratique artistique qui sont problématiques pour la théorie du sens commun comprennent : a) la matière dont l'œuvre est faite, si la peinture est un faux, pour que, par exemple, nos jugements sur la valeur 
artistique se transforment lorsque nous découvrons que les Disciples d'Emmaüs est de van Meegeren et non de Vermeer; b) notre intérêt, cultivé par les conservateurs de musée, pour la manière dont les objets d'art sont produits - par exemple, l'exposition, à côté des peintures, des premiers croquis des Demoiselles d'Avignon de Picasso, et l'attention portée au fait que Vermeer a utilisé une camera obscura pour plusieurs de ses toiles; et c) les difficultés que rencontrent ceux qui possèdent quelque chose comme la théorie du sens commun lorsqu'ils tentent de comprendre et d'apprécier plusieurs œuvres de l'art moderne tardif. Ces questions se posent non seulement pour l'art visuel, mais aussi pour le théâtre, la danse, le cinéma, la littérature, la musique, et les autres formes d'art. Pour les «empiristes esthétiques" qui adhèrent à la vision appréciative du sens commun, notre pratique est simplement erronée lorsqu'elle considère que de tels aspects de l'histoire de la production d'objets d'art sont eux-mêmes susceptibles d'être appréciés en tant qu'œuvres : ils ne concernent que les éléments sociologiques, psychologiques et artistico-historiques des produits de la création artistique.

Je réponds à ces questions de deux façons similaires. Premièrement, dans les chapitres 2 et 3, je donne un fondement plus solide aux récents travaux en épistémologie de l'art qui s'attaquent à la manière d'apprécier de l'esthétique empiriste. J'examine alors de quelle façon la connaissance historique de la création du produit d'une activité artistique repose sur l'appréciation de l'œuvre créée. Ce faisant, j'introduis le terme de "centre d'appréciation » [focus of appreciation] pour renvoyer au produit d'une performance artistique créatrice, dans la mesure où ce produit est considéré comme pertinent pour l'appréciation de l'œuvre ainsi créée. M'appuyant sur les travaux de Currie, Dutton, Danto, Levinson, Wollheim, Blinkley et autres, je soutiens que le centre d'appréciation devrait être considéré comme ayant une certaine structure, et que nous pouvons comprendre cette structure sans situer le véhicule artistique dans le contexte de l'histoire de sa production. Des œuvres d'art sont créées parce que quelque chose est fait dans un contexte où cette action est vue comme produisant autre chose. Par exemple, quelqu'un applique de la peinture à l'huile sur une toile, et cela est perçu comme la production d'un tableau auquel diverses propriétés représentationnelles, expressives et formelles peuvent être attribuées par les spectateurs. J'appelle la première action «manipuler un médium-véhicule» et le produit créé le "véhicule artistique ». Je nomme la seconde "exprimer un énoncé artistique ", une expression fourretout qui rassemble l'ensemble des propriétés largement «signifiantes » que nous attribuons aux produits de l'activité artistique dans notre appréhension critique de ces produits. Les éléments qui composent l'énoncé artistique exprimé par l'intermédiaire d'un véhicule artistique peuvent inclure le fait d'être une forme de représentation, d'avoir une valeur expressive particulière, ou de rendre manifeste quelque propriété formelle ou matérielle, de même que l'ensemble des significations d'ordre supérieur que nous attribuons au véhicule en vertu de ces significations élémentaires. Utiliser un médium à titre 
de véhicule se présente comme l'expression d'un énoncé artistique, parce que le contexte à l'intérieur duquel le produit de la première action est perçu fournit des normes interprétatives ou des formes de compréhension partagées qui nous permettent de le saisir ainsi. Reprenant l'expression de Margolis, je donne à ces formes de compréhension partagées le nom de «médium artistique ». Ainsi, le produit d'une performance artistique créatrice constitue pour les fins de l'appréciation — du "centre d'appréciation » — un énoncé artistique exprimé par un véhicule artistique en vertu d'un médium artistique.

Je soutiens donc, en m'appuyant encore une fois sur le travail des philosophes mentionnés plus haut, que nous ne pouvons identifier le centre d'appréciation que si nous situons le produit manifeste - le véhicule artistique dans l'histoire d'une production. D'ailleurs, cela peut même s'avérer nécessaire pour identifier le véhicule - comme c'est le cas avec le film contemporain d'animation, et, pour différentes raisons, dans l'art moderne tardif. Cela nous aide à comprendre ce qui se passe par ailleurs lorsque des spectateurs compétents ne parviennent pas à "saisir» l'art moderne tardif — lorsque, par exemple, ils prennent le véhicule artistique dans une œuvre comme la Fontaine de Duchamp pour l'objet exposé dans la galerie et le médium artistique pour celui que l'on retrouve normalement dans les œuvres de sculpture.

Dans les sept autres chapitres, j'élabore et défends une position particulière quant au statut ontologique de l'œuvre d'art, une position qui, je pense, constitue la meilleure option théorique compte tenu de la contrainte pragmatique, dès lors que nous acceptons la critique de l'esthétique empiriste développée dans les deux chapitres précédents. Selon cette conception, que je nomme «théorie de la performance ", des œuvres d'art de toutes sortes peuvent être identifiées, non aux produits des activités créatrices des artistes, mais avec ces activités créatrices elles-mêmes, qui sont complétées par ces produits : l'œuvre est une performance créatrice qui réside dans les activités intentionnelles d'un médium véhiculaire par lesquelles un centre d'appréciation particulier se trouve précisé. Le cheval de bataille du long argument en faveur de la théorie de la performance n'est pas l'ontologie du sens commun mais un "contextualisme " qui a été considéré comme la réponse ontologique naturelle à l'abandon de l'esthétique empiriste. Les contextualistes conçoivent une œuvre comme le produit contextualisé d'un acte de production artistique, et un centre d'intérêt comme la compréhension nécessitant la connaissance de certains aspects de ces actes, dont l'identité, en tant qu'œuvre, comprend en quelque sorte l'histoire de la production. Pour le contextualiste, une œuvre est un véhicule artistique en tant qu'elle est créée dans un contexte artistico-historique particulier : par exemple, Jerrold Levinson affirme que les œuvres musicales ne sont pas de pures structures de sons (ou que les sons/performances sont de purs moyens) mais constituent des structures en tant qu'elles sont «indiquées» dans un tel contexte, tandis que Danto propose une théorie contextualiste des œuvres d'art visuel reproduisant une théorie contextualiste préalablement appliquée aux œuvres d'art littéraires. 
L'argument proposé pour la théorie de la performance, et contre le contextualisme, est "cumulatif» en ce sens que les considérations individuelles invoquées acquièrent plus de poids lorsqu'il devient clair qu'elles prennent place dans un cadre théorique plus large, ce qui nous permet de réfléchir sur la pratique artistique et de discourir sur l'art. Ce cadre théorique devient pleinement évident seulement au dernier chapitre, après que la notion de performance artistique, centrale pour la théorie de la performance, a été expliquée et bien détaillée. L'analyse de la structure raffinée du centre d'appréciation au chapitre 3 constitue une étape cruciale dans le développement de mon argument. Elle rend vaine l'intérêt de l'empiriste pour une distinction entre propriétés artistiques et propriétés artistico-historiques. Mais cela nous conduit aussi à nous demander s'il existe en principe une raison d'exclure de notre appréciation des œuvres les éléments d'origine [aspects of provenance] non déterminants pour les propriétés du centre d'appréciation.

Au chapitre 4, je soutiens que la logique même de l'argument contre l'empirisme, tel qu'élaboré à partir du centre d'appréciation, met en question la tentative contextualiste visant à exclure de l'épistémologie de l'art de tels éléments d'origine. Je défie le contextualiste de se tourner, en vertu d'un principe, vers les aspects qui nous intéressent dans la performance créatrice d'un artiste, sans que cela ne laisse de trace sur le centre [d'appréciation] qui en résulte - par exemple, les premiers brouillons d'une œuvre, une preuve, déterminable seulement aux rayons $\mathrm{X}$, qu'on a repeint sur une toile, ou la preuve que certains aspects de notre centre d'appréciation sont non intentionnels ou accidentels. Relativement à ce dernier type d'exemple, je soutiens que la théorie de la performance nous permet de contourner les désaccords opposant les théories intentionnaliste et non intentionnaliste de l'interprétation, dans la mesure où de tels désaccords supposent que les intentions artistiques peuvent porter sur l'appréciation et la valeur artistiques seulement si elles déterminent l'énoncé artistique exprimé à l'aide du véhicule artistique. Dès lors que nous reconnaissons les différentes manières selon lesquelles notre intérêt critique à propos d'une œuvre d'art nécessite que nous tenions compte de la performance créatrice, le fardeau de la preuve réside entre les mains du contextualiste qui doit démontrer pourquoi nous devrions malgré tout considérer l'œuvre comme un produit contextualisé.

Au chapitre 5, je développe un argument «modal» complétant l'argument du chapitre 4 , mais qui fournit aussi une raison particulière de questionner le caractère approprié du contextualisme. L'objection initiale contre l'ontologie contextualiste, comme celle de Levinson, consiste à dire qu'en déterminant certains types d'éléments originaux [aspects of provenance] constituant toute œuvre, cette ontologie manque de ressources pour rendre compte de ce que j'appelle le «fonctionnement relatif de la modalité » [work-relativity of modality] — le fait que les éléments d'origine portent sur nos jugements modaux avec une force variable reflétant notre perception de ce qui doit être apprécié dans une œuvre donnée. En fait, je soutiens que nos intuitions 
modales au sujet des œuvres reprennent nos intuitions modales à propos de performances particulières là où nos centres [d'appréciation] sont précisés. La théorie de la performance, en identifiant les œuvres à des performances particulières partageant la propriété générale d'être l'objet de précisions du centre d'appréciation, peut aisément s'accommoder de ce fait. Même une version révisée du contextualisme, identifiant les œuvres avec les centres de l'œuvre [work-focuses], précisés par une performance de manipulation particulière, pourrait être exposée à cette critique, puisque nous ne pouvons rien dire sur ce qui distingue ces performances.

Ces arguments pour la théorie de la performance sont étayés au cours de l'élaboration détaillée de la théorie au chapitre 7 . Cette élaboration est tributaire de l'évaluation critique, au chapitre 6 , des difficultés rencontrées par l' 'hypothèse de l'action-type » de Gregory Currie, laquelle identifie les œuvres d'art avec des types d'action plutôt qu'avec des performances symboliques [token performances]. Pour Currie, une œuvre est un type d'action lorsqu'une structure particulière est découverte par une «voie heuristique » particulière. Je soutiens, contre Currie : a) que la notion de «voie heuristique» ne peut pas remplir la fonction ontologique qu'on lui accorde; b) que l'application de la notion de «structure" pour l'ontologie de l'art est limitée; c) qu'une interprétation réaliste ou nominaliste des «types d'action» ne peut nous fournir une ontologie de l'art appropriée; et d) que Currie ne parvient pas à rendre compte du fonctionnement relatif de la modalité [work-relativity of modality]. En élaborant la théorie de la performance, je ne parle pas de «découvrir une structure ", mais de "préciser un centre d'appréciation ". En individuant les œuvres comme des performances ainsi identifiées, je fais intervenir, conformément à la notion de fonctionnement relatif de la modalité, l'idée d'une caractérisation adéquate des actions intentionnelles lorsqu'un centre d'appréciation a été précisé - une caractérisation adéquate de ce qui a été accompli dans une performance créatrice particulière. Une œuvre, en tant que performance, comporte tout simplement ces actions accomplies par le ou les artistes, ainsi que les aspects du contexte de la performance que nous pouvons inclure dans une caractérisation adéquate des actions intentionnelles qui constituent le véhicule artistique de l'œuvre.

Plus loin je soutiens que les œuvres, en tant que performances spécifiques, ne doivent pas être identifiées avec des "actions élémentaires " qui peuvent avoir essentiellement leur moment de réalisation, mais avec ce que je nomme des "accomplissements » [doings], des exemples d'événements admettant les formes de variation des propriétés modales nécessaires au fonctionnement relatif de la modalité. Je soutiens que les accomplissements sont nécessaires pour donner une signification à une bonne partie de notre discours modal à propos des événements, et que les œuvres d'art sont des accomplissements d'un genre particulier. Pour apprécier une œuvre il faut apprécier ce qui a été accompli, et, en évaluant des affirmations modales au sujet des œuvres, on se demande si cela peut avoir été fait en de telles circonstances. 
Notre sentiment à propos de ce que c'est est capté par ce que nous tenons pour une caractérisation adéquate des actions intentionnelles du médium véhiculaire en vertu de laquelle le centre d'appréciation se trouve précisé. Un accomplissement particulier est réalisé par un ensemble particulier d'exemples d'actions-types [a particular set of simpler action-tokens] dans le monde réel, mais peut [aussi] être réalisé par différents exemples d'actions-types symboliques dans d'autres mondes possibles.

Deux considérations supplémentaires favorisant la théorie de la performance plutôt que le contextualisme sont présentées au chapitre 8. D'abord, une objection contextualiste courante à l'encontre des ontologies de l'événement artistique consiste à dire que de telles ontologies rectifient radicalement notre discours ordinaire sur les œuvres d'art et nécessitent une distinction entre parler de l'œuvre et parler d'un certain élément propre à l'œuvre (par exemple le centre d'appréciation). [Or] cette objection se retourne contre le contextualiste; lorsqu'on se demande s'il est susceptible de recevoir une objection similaire, on découvre une instabilité fondamentale dans sa position. Cela apparaît de la manière la plus évidente dans la récente critique que Julian Dodd adresse au type de contextualisme défendu par Levinson. Si une époque ou tout autre paramètre temporel indiqué [indexed] est représenté comme un aspect constitutif d'une œuvre comme c'est le cas dans la théorie de la «structure indiquée ", alors, soutient Dodd, des œuvres pourrait être des entités s'apparentant à des événements, puisque seules de telles entités peuvent être marquées dans le temps ou avoir des propriétés temporellement infléchies [inflected] comme éléments constitutifs. Si, d'un autre côté, les époques ou propriétés temporellement indiquées ne sont pas considérées comme des éléments constitutifs des œuvres des contextualistes, alors les œuvres doivent être pensées comme des structures individuées en fonction de l'époque de leur occurrence. Par conséquent, le contextualisme est instable, il s'effondre lorsqu'il est soumis à un examen minutieux, soit par une certaine forme d'ontologie décontextualisée, ou bien par une ontologie de l'événement comme la théorie de la performance. Dodd prend cela pour un argument favorisant le rétablissement d'une conception structuraliste des œuvres musicales et littéraires. $\mathrm{Si}$, toutefois, nous rejetons le structuralisme, nous pouvons répondre en acceptant une ontologie de l'événement comme la théorie de la performance. Nous pourrions alors adapter les implications «révisionnistes» d'une telle ontologie en nous dotant d'une distinction de principe entre parler des œuvres et parler des éléments qui constituent le centre [d'appréciation] de l'œuvre.

Une deuxième considération favorisant la théorie de la performance est implicite dans les sections précédentes du chapitre 8 , où je soutiens que le contextualisme ne peut pas adéquatement rendre compte des distinctions cruciales qui doivent être faites entre les œuvres de l'art moderne tardif et qu'il est aussi incapable d'expliquer le traitement que nous réservons aux œuvres contrefaites. La théorie de la performance, selon moi, est en bien meilleure position pour rendre compte de ces dimensions de notre pratique artistique. Cet argument 
pour la théorie de la performance est développé au chapitre 9 , où une analyse détaillée de la manière dont les événements-performances - comme les performances d'œuvres musicales ou théâtrales - sont créés et appréciés comme étant des œuvres d'art. La théorie de la performance nous fournit un cadre plus large dans lequel nous pouvons inclure cette analyse des événements-performances, de telle sorte que nous soyons en mesure de faire d'importantes distinctions entre les œuvres de l'art moderne tardif, ainsi qu'entre différents genres d'événements-performances dans les arts [en général].

Dans le dernier chapitre, je complète la critique de la théorie de l'art du sens commun entamée au premier chapitre. Premièrement, j'indique de quelle manière nous serions en mesure de définir une "œuvre d'art» comme un type de performance, en distinguant ainsi les œuvres d'art des autres performances créatrices de véhicules qui expriment des contenus en vertu de formes de compréhension communes. Je propose [ainsi] de considérer qu'une œuvre d'art est une performance exprimant un contenu à l'aide d'un véhicule, par un "médium artistique ", [c'est-à-dire] un système de formes de compréhension dont l'expression est commune au système du monde de l'art. Le système du monde de l'art, quant à lui, est un système à l'intérieur duquel les formes de compréhension dont l'expression est commune facilitent l'expression d'un contenu par des véhicules qui remplissent des fonctions symboliques «esthétiques » au sens de Goodman. Une « œuvre d'art» est définie de manière procédurale, en référence à une performance s'appuyant intentionnellement sur un système préétabli de formes de compréhension évidentes [articulatory understandings], et fonctionnellement, dans la mesure où c'est en référence à un certain type de fonctionnement symbolique qu'un système de formes de compréhension évidentes est considéré comme un médium artistique.

Deuxièmement, je défends une théorie de la valeur artistique qui à la fois embrasse [l'ensemble] des types d'expérience d'évaluation [experiential values] que célèbre l'empiriste et permet aussi aux œuvres de posséder une valeur artistique, en vertu de leur statut de performances ou d' " accomplissements " [doings]. Je m'oppose ici à ce que j'appelle "l'empirisme éclairé ", qui admet d'une part la critique contextualiste des théories empiristes de l'appréciation, mais qui maintient de l'autre une théorie empiriste de la valeur artistique. Cela complète aussi l'élaboration d'un cadre philosophique plus large pour réfléchir sur les œuvres d'art, - leur être, leur être-appréciées et leur êtreévaluées - fondé sur la théorie de la performance. Mon argument cumulatif fait alors intervenir la "contrainte pragmatique ", selon laquelle nous devons évaluer les propositions ontologiques en fonction de leur adéquation à un cadre philosophique plus étendu, qui rend notre pratique signifiante en tant que cette pratique est «codifiée» par la réflexion rationnelle. C'est sa place à l'intérieur de la considération philosophique générale de notre pratique artistique, élaborée dans mon livre, - un cadre qui montre tant les continuités que les discontinuités saillantes entre l'art traditionnel et l'art moderne, et entre différentes disciplines artistiques -, qui nous procure la mesure 
ultime de la théorie de la performance comme ontologie de l'art. Le fardeau de la preuve repose alors sur le contextualiste qui doit proposer une ontologie contextualiste surpassant la théorie de la performance ainsi comprise.

\section{Le statut de l'œuvre d'art comme événement chez David Davies}

\section{ROGER POUIVET}

Université de Nancy 2 et Archives Poincaré (CNRS)

Roger.Pouivet@univ-nancy2.fr

[Peter Hutchinson] filled some plastic bags with gas and pieces of rotten calabash or something of the sort ... threw the whole business into the ocean... An underwater photographer took pictures of the installation... Genius and process - process and genius!

Tom Wolfe, The Painted Word

1.

Il existe deux types d'ontologie de l'art. Le premier consiste à utiliser les concepts clés de l'ontologie, les notions d'objet, de propriété, d'identité, etc., afin d'expliciter le mode d'existence des œuvres. Une telle ontologie, en quelque sorte appliquée, examine la question de l'authenticité des oeuvres, des modalités de leur exposition, de leur restauration, de la relation à leurs exécutions, à leurs enregistrements, etc. Elle ne recherche pas une conception ontologique générale et unifiée de ce en quoi consiste être une œuvre d'art. Elle est nécessairement pluraliste. On en trouve un bon exemple chez Stephen Davies ${ }^{1}$. David Davies pratique l'autre type, tout comme Wolterstorff, Currie, Zemach. Cette ontologie générale et unifiée est dualiste ou moniste, selon qu'elle affirme l'existence d'œuvres relevant de deux catégories ontologiques (les entités singulières et les entités instanciables), ou d'une seule catégorie. David Davies n'affirme pas simplement que certaines œuvres, particulièrement les œuvres relevant de «l'art contemporain» — celles de Duchamp, Beuys ou Acconci sont mieux décrites en termes d'événements que d'objets ou de produits, que notre seul espoir de les apprécier à leur juste valeur artistique ou esthétique suppose de les tenir pour des événements, comme on pourrait le faire dans une ontologie du premier type. Il affirme que toutes les œuvres d'art, sans exception, sont des performances, c'est-à-dire des événements.

\section{2.}

La défense par David Davies d'une ontologie événementielle des œuvres d'art fera date. La lecture d'un livre aussi superbement maîtrisé pourrait (presque) 